\title{
THE CO-STABILITY MANIFOLD OF A TRIANGULATED CATEGORY
}

\author{
PETER JØRGENSEN \\ School of Mathematics and Statistics, Newcastle University, Newcastle upon Tyne NE1 7RU, United Kingdom \\ e-mail: peter.jorgensen@ncl.ac.uk \\ and DAVID PAUKSZTELLO \\ Institut für Algebra, Zahlentheorie und Diskrete Mathematik, Fakultät für Mathematik und Physik, Leibniz \\ Universität Hannover, Welfengarten 1, 30167 Hannover, Germany \\ e-mail:pauk@math.uni-hannover.de
}

(Received 30 September 2011; accepted 21 December 2011; first published online 2 August 2012)

\begin{abstract}
Stability conditions on triangulated categories were introduced by Bridgeland as a 'continuous' generalisation of t-structures. The set of locally-finite stability conditions on a triangulated category is a manifold that has been studied intensively. However, there are mainstream triangulated categories whose stability manifold is the empty set. One example is $\mathrm{D}^{\mathrm{c}}\left(k[X] /\left(X^{2}\right)\right)$, the compact derived category of the dual numbers over an algebraically closed field $k$. This is one of the motivations in this paper for introducing co-stability conditions as a 'continuous' generalisation of co-t-structures. Our main result is that the set of nice co-stability conditions on a triangulated category is a manifold. In particular, we show that the co-stability manifold of $\mathbb{D}^{\mathfrak{c}}\left(k[X] /\left(X^{2}\right)\right)$ is $\mathbb{C}$.
\end{abstract}

2010 Mathematics Subject Classification. 18E30.

1. Introduction. Triangulated categories are useful in several branches of mathematics, and stability conditions are an important tool for their study introduced by Bridgeland in [4]. Stability conditions are 'continuous' generalisations of bounded t-structures and the main result of [4] is that on an essentially small triangulated category, the set of stability conditions which satisfy the technical condition of local finiteness is a manifold. This 'stability manifold' is divided into subsets corresponding to bounded t-structures in the category.

However, there are mainstream triangulated categories for which the stability manifold is the empty set. An example is $\mathrm{D}^{\mathrm{c}}\left(k[X] /\left(X^{2}\right)\right)$, the compact derived category of the dual numbers over an algebraically closed field $k$. This is our first motivation for introducing the 'mirror' notion of co-stability conditions and proving the following main theorem.

THEOREM A. Let T be a triangulated category satisfying the conditions in setup 1.1 below. Then the set of co-stability conditions on $\mathrm{T}$ which satisfy the technical condition in Definition 8.1 is a topological manifold.

Indeed, the 'co-stability manifold' of the category $\mathrm{D}^{\mathrm{c}}\left(k[X] /\left(X^{2}\right)\right)$ which exists by Theorem A is non-trivial: 
THEOREM B. Let $k$ be an algebraically closed field and consider $\mathrm{D}^{\mathrm{c}}\left(k[X] /\left(X^{2}\right)\right)$. Its stability manifold is the empty set and its co-stability manifold is $\mathbb{C}$.

The co-stability manifold of a triangulated category is divided into subsets corresponding to bounded co-t-structures in the category; see Remark 8.5. Recall that co-t-structures are, in a sense, a mirror image of t-structures. They were introduced independently in [7, Definition 2.4] and [3, Definition 1.1.1] where they were called weight structures; see Definition 2.1. They have recently been the focus on considerable interest, see for instance $[\mathbf{1}, \mathbf{3}, \mathbf{5}, \mathbf{6}, \mathbf{7}]$.

Equivalently, the co-stability manifold is divided into subsets corresponding to silting subcategories as defined in [1, Definition 2.1], because these are in bijection with bounded co-t-structures by [6, Corollary 4.7]. These observations are our second motivation for introducing co-stability conditions.

Relation to Bridgeland's paper [4]. Recall that a stability condition is a pair $(Z, \mathscr{P})$ where $Z: \mathrm{K}_{0}(\mathrm{~T}) \rightarrow \mathbb{C}$ is a homomorphism and $\mathscr{P}$ a so-called slicing consisting of certain subcategories $\mathscr{P}(\varphi)$ for $\varphi \in \mathbb{R}$. It is required that $Z(p)=m(p) \exp (i \pi \varphi)$ with $m(p)>0$ for $p \in \mathscr{P}(\varphi)$ with $p \nsucceq 0$.

We define co-stability conditions analogously, replacing the slicing $\mathscr{P}$ with a coslicing $\mathscr{Q}$; this notion is defined in Section 3. Some other parts of what we do are also closely inspired by [4] as we shall point out along the way.

However, the passage from stability conditions to co-stability conditions is nontrivial. It is governed by a 'looking glass principle' (a term coined in [2]): Some results on stability conditions have mirror versions for co-stability conditions, but others do not and translation is rarely mechanical. In fact, this is already true of the passage from $t$-structures to co-t-structures and means that our proofs are different from those in [4].

Further remarks and setup. We have chosen only to define the co-stability manifold for triangulated categories that are Krull-Schmidt with finitely generated $\mathrm{K}_{0}$-group. This covers the examples we have in mind from representation theory, ensures that the co-stability manifold is finite dimensional and makes the theory less technical.

However, everything in Sections 2 and 3 works for any essentially small triangulated category, with the exception of Remark 3.8. It should also be possible to develop the rest of the theory in this generality, at the cost of being more technical along the lines of $[4$, Sections 6 and 7].

The paper is organised as follows: Section 2 recapitulates the definition of co-tstructures. Section 3 defines co-slicings in triangulated categories. Section 4 turns the set of co-slicings into a metric space. Section 5 defines co-stability functions and the split Harder-Narasimhan property. Section 6 defines co-stability conditions and proves a crucial separation result in Proposition 6.2. Section 7 has two technical lemmas. Section 8 proves an equally crucial deformation result in Proposition 8.4; Theorem $\mathrm{A}$ is a consequence which appears as Theorem 8.3. Section 9 remarks that, like the stability manifold, the co-stability manifold admits commuting group actions of Aut(T) and $\widetilde{G L}^{+}(2, \mathbb{R})$. Section 10 proves Theorem $\mathrm{B}$ which is a special case of Theorem 10.1 . Section 11 gives an example explaining why the technical condition in Definition 8.1 is necessary for Proposition 8.4 and hence for Theorem A.

We end Section 1 with some notation. When $\mathrm{T}$ is a category, $\mathrm{T}(-,-)$ is a shorthand for $\operatorname{Hom}_{\top}(-,-)$. The suspension functor of a triangulated category is denoted by $\Sigma$. Distinguished triangles are sometimes written $t^{\prime} \longrightarrow t \longrightarrow t^{\prime \prime} \leadsto t^{\prime}$; the wiggly arrow is short for a morphism $t^{\prime \prime} \rightarrow \Sigma t^{\prime}$. 
When we say that an additive category is Krull-Schmidt, we mean that it has split idempotents, that each object is the direct sum of finitely many indecomposable objects and that each indecomposable object has local endomorphism ring. The Krull-Schmidt theorem then implies that the indecomposable direct summands of a given object are determined up to isomorphism. The prefix ind denotes the class of indecomposable objects in an additive category.

We always assume that subcategories are strict, that is, closed under isomorphisms. In other words, if $a$ is an object of a subcategory and $a \cong a^{\prime}$ in the ambient category, then $a^{\prime}$ is also an object of the subcategory. The symbol $\perp$ sends full subcategories of an additive category $\mathrm{T}$ to full subcategories as follows:

$$
\mathrm{A}^{\perp}=\{t \in \mathrm{T} \mid \mathrm{T}(\mathrm{A}, t)=0\}, \quad{ }^{\perp} \mathrm{B}=\{t \in \mathrm{T} \mid \mathrm{T}(t, \mathrm{~B})=0\} .
$$

Each of our categorical closure operations is understood as producing full subcategories. In particular, in an additive category, add denotes closure under finite direct sums and direct summands, and in a triangulated category, ()$^{-}$denotes closure under extensions, while ( $)^{+}$denotes closure under extensions and direct summands.

The split Grothendieck group of an additive category $T$ is denoted by $K_{0}^{\text {split }}(T)$. It is the commutative group with a generator $[t]$ for each object $t \in \mathrm{T}$ and a relation $[t]=\left[t^{\prime}\right]+\left[t^{\prime \prime}\right]$ for each isomorphism $t \cong t^{\prime} \oplus t^{\prime \prime}$ in $\mathrm{T}$.

The Grothendieck group of a triangulated category $T$ is denoted by $\mathrm{K}_{0}(\mathrm{~T})$. It is the commutative group with a generator $[t]$ for each object $t \in \mathrm{T}$ and a relation $[t]=\left[t^{\prime}\right]+\left[t^{\prime \prime}\right]$ for each distinguished triangle $t^{\prime} \longrightarrow t \longrightarrow t^{\prime \prime} \leadsto t^{\prime}$ in $\mathrm{T}$.

Setup 1.1. From now on, $T$ is an essentially small triangulated category that is Krull-Schmidt and has finitely generated $\mathrm{K}_{0}(\mathrm{~T})$.

2. Co-t-structures. This section recalls the definition of co-t-structures and two useful properties. The definition is due independently to [3, Definition 1.1.1] and [7, Definition 2.4]; we have tweaked it slightly for reasons of symmetry.

Definition 2.1. A co-t-structure in $T$ is a pair $(A, B)$ of full subcategories closed under direct sums and summands satisfying the following conditions:

(i) $\Sigma^{-1} \mathrm{~A} \subseteq \mathrm{A}$ and $\Sigma \mathrm{B} \subseteq \mathrm{B}$.

(ii) $\mathrm{T}(\mathrm{A}, \mathrm{B})=0$.

(iii) For each object $t \in \mathrm{T}$, there is a distinguished triangle $a \rightarrow t \rightarrow b$ with $a \in \mathrm{A}$, $b \in \mathrm{B}$.

The co-heart is $\mathrm{C}=\mathrm{A} \cap \Sigma^{-1} \mathrm{~B}$.

The co-t-structure is called bounded if

$$
\bigcup_{j \in \mathbb{Z}} \Sigma^{j} \mathrm{~A}=\bigcup_{j \in \mathbb{Z}} \Sigma^{j} \mathrm{~B}=\mathrm{T} .
$$

REMARK 2.2. Note that if we replace (i) by the conditions $\Sigma \mathrm{A} \subseteq \mathrm{A}$ and $\Sigma^{-1} \mathrm{~B} \subseteq \mathrm{B}$, then we get the definition of a t-structure.

The following two results were proved in [3, Proposition 1.5.6 and Theorem 5.3.1]. We restate them for the convenience of the reader. Note that Proposition 2.3 is the co-t-structure analogue of [4, Lemma 3.2]. 
Proposition 2.3. Let (A, B) be a bounded co-t-structure in $\mathrm{T}$ with co-heart $\mathrm{C}$. For each object $t \in \mathrm{T}$, there is a diagram

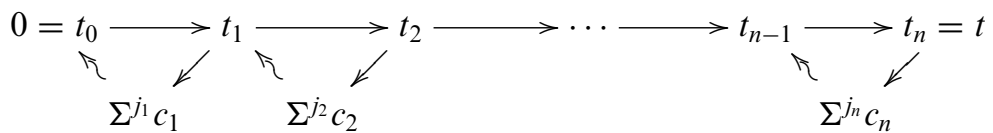

consisting of distinguished triangles, where $n \geq 1, c_{m} \in \mathrm{C}$ for each $m$, and $j_{1}<j_{2}<\cdots<$ $j_{n}$.

Proposition 2.4. Let (A, B) be a bounded co-t-structure in $\mathrm{T}$ with co-heart $\mathrm{C}$. There is an isomorphism

$$
\mathrm{K}_{0}^{\text {split }}(\mathrm{C}) \stackrel{\sim}{\rightarrow} \mathrm{K}_{0}(\mathrm{~T})
$$

given by $[c] \mapsto[c]$.

The inverse is $[t] \mapsto \sum_{m}\left[\Sigma^{j_{m}} c_{m}\right]$ where the objects $\Sigma^{j_{m}} c_{m}$ come from a diagram as in Proposition 2.3; this sum determines a well-defined element of $\mathrm{K}_{0}^{\mathrm{split}}(\mathrm{C})$.

3. Co-slicings. This section introduces co-slicings. They are a mirror image of the slicings of [4, Definitions 3.3].

Definition 3.1. A co-slicing $\mathscr{Q}$ of $\mathrm{T}$ is a collection of full subcategories $\mathscr{Q}(\varphi)$ closed under direct sums and summands, indexed by $\varphi \in \mathbb{R}$ and satisfying the following conditions:

(i) $\mathscr{Q}(\varphi+1)=\Sigma \mathscr{Q}(\varphi)$.

(ii) $\varphi_{1}<\varphi_{2} \Rightarrow \mathrm{T}\left(\mathscr{Q}\left(\varphi_{1}\right), \mathscr{Q}\left(\varphi_{2}\right)\right)=0$.

(iii) For each object $t \in \mathrm{T}$, there is a diagram

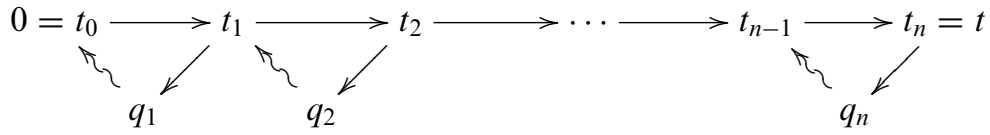

consisting of distinguished triangles, where $n \geq 1, q_{i} \in \mathscr{Q}\left(\varphi_{i}\right)$, and $\varphi_{1}<\cdots<$ $\varphi_{n}$.

Note that (i) and (ii) are continuous versions of (i) and (ii) in Definition 2.1, while (iii) is a continuous version of Proposition 2.3.

LEMMA 3.2. Let $\mathscr{Q}$ be a co-slicing of $\mathrm{T}$ and consider the diagram from Definition 3.1(iii). For each $j$, there is an obvious morphism $t_{j} \rightarrow t$ which we complete to a distinguished triangle

$$
t_{j} \rightarrow t \rightarrow e_{j}
$$

Then the objects $e_{j}$ sit in a diagram

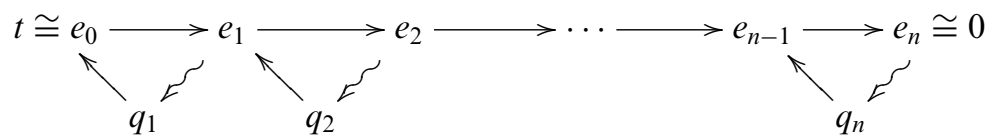


consisting of distinguished triangles.

This diagram and the one from Definition 3.1(iii) show

$$
t_{j} \in\left(\mathscr{Q}\left(\varphi_{1}\right) \cup \cdots \cup \mathscr{Q}\left(\varphi_{j}\right)\right)^{-}, \quad e_{j} \in\left(\mathscr{Q}\left(\varphi_{j+1}\right) \cup \cdots \cup \mathscr{Q}\left(\varphi_{n}\right)\right)^{-} .
$$

Recall that ( ) ${ }^{-}$denotes closure under extensions.

Proof. It is clear that $e_{0} \cong t$ and $e_{n} \cong 0$. To get the distinguished triangles forming the diagram in the lemma, apply the octahedral axiom to the composable morphisms $t_{j-1} \rightarrow t_{j} \rightarrow t$ for each value of $j$ to get the following $3 \times 3$ diagrams of distinguished triangles.

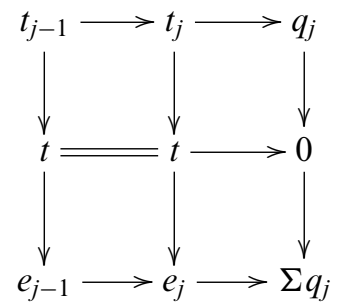

Definition 3.3. Let $\mathscr{Q}$ be a co-slicing of T. For $I \subseteq \mathbb{R}$, we define a full subcategory of $\mathrm{T}$ by

$$
\mathscr{Q}(I)=\left(\bigcup_{\varphi \in I} \mathscr{Q}(\varphi)\right)^{+}
$$

Recall that ()$^{+}$denotes closure under extensions and direct summands.

As a shorthand, we combine this with inequality signs in an obvious way; for instance, $\mathscr{Q}(<a)=\mathscr{Q}(]-\infty, a[)$.

Definition 3.1(i) implies

$$
\Sigma \mathscr{Q}(I)=\mathscr{Q}(\Sigma I)
$$

where $\Sigma I=\{i+1 \mid i \in I\}$. Definition 3.1(iii) implies that $\mathscr{Q}(\mathbb{R})=\mathrm{T}$.

LEMMA 3.4. Let $\mathscr{Q}$ be a co-slicing of $\mathrm{T}$. For $a \leq b$ in $\mathbb{R}$, we have

$$
\left.\left.{ }^{\perp} \mathscr{Q}(>b) \cap \mathscr{Q}(\leq a)^{\perp}=\mathscr{Q}(] a, b\right]\right) .
$$

Proof. The inclusion $\supseteq$ is clear from Definition 3.1(ii).

To see $\subseteq$, let

$$
t \in{ }^{\perp} \mathscr{Q}(>b) \cap \mathscr{Q}(\leq a)^{\perp}
$$

and consider the diagrams from Definition 3.1(iii) and Lemma 3.2. The lemma implies

$$
t_{j} \in \mathscr{Q}\left(\left[\varphi_{1}, \varphi_{j}\right]\right) \text { and } e_{j} \in \mathscr{Q}\left(\left[\varphi_{j+1}, \varphi_{n}\right]\right)
$$


If $b<\varphi_{1}$, then equation (3) implies that $t=t_{n} \in \mathscr{Q}(>b)$. By equation (2), it follows that $\mathrm{T}(t, t)=0$ so $t \cong 0$ and $t \in \mathscr{Q}(] a, b])$ is clear.

If $\varphi_{1} \leq b$, then let $1 \leq \ell \leq n$ be maximal with $\varphi_{\ell} \leq b$. Equation (3) implies $e_{\ell} \in$ $\mathscr{Q}(>b)$ and by equation (2), it follows that $\mathrm{T}\left(t, e_{\ell}\right)=0$. Hence, the distinguished triangle $\Sigma^{-1} e_{\ell} \rightarrow t_{\ell} \rightarrow t$ is split and we have $t_{\ell} \cong t \oplus t^{\prime}$ where $t^{\prime}=\Sigma^{-1} e_{\ell}$. Truncating the diagram from Definition 3.1(iii) gives

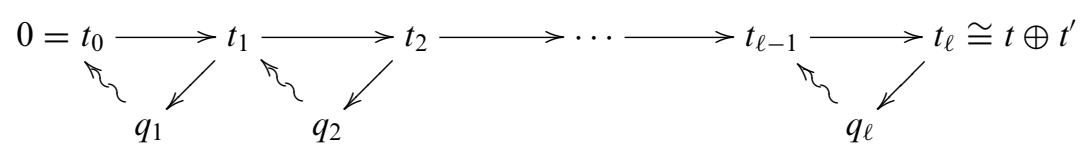

with $q_{j} \in \mathscr{Q}\left(\varphi_{j}\right)$ and $\varphi_{1}<\cdots<\varphi_{\ell} \leq b$.

If $a<\varphi_{1}$, then diagram (4) shows $\left.\left.t \oplus t^{\prime} \in \mathscr{Q}(] a, b\right]\right)$ whence $\left.\left.t \in \mathscr{Q}(] a, b\right]\right)$ as desired.

If $\varphi_{1} \leq a$, then let $1 \leq m \leq \ell$ be maximal with $\varphi_{m} \leq a$. By Lemma 3.2 applied to diagram (4) there is a distinguished triangle

$$
t_{m} \rightarrow t \oplus t^{\prime} \rightarrow f_{m}
$$

with

$$
\left.\left.f_{m} \in\left(\mathscr{Q}\left(\varphi_{m+1}\right) \cup \cdots \cup \mathscr{Q}\left(\varphi_{\ell}\right)\right)^{-} \subseteq \mathscr{Q}\left(\left[\varphi_{m+1}, \varphi_{\ell}\right]\right) \subseteq \mathscr{Q}(] a, b\right]\right) .
$$

Equation (3) implies $t_{m} \in \mathscr{Q}(\leq a)$ and by equation (2), it follows that $\mathrm{T}\left(t_{m}, t\right)=0$, so the distinguished triangle is isomorphic to the direct sum of distinguished triangles $0 \rightarrow t \stackrel{=}{\rightarrow} t$ and $t_{m} \rightarrow t^{\prime} \rightarrow f_{m}^{\prime}$. Hence, $f_{m} \cong t \oplus f_{m}^{\prime}$ and so $\left.\left.t \in \mathscr{Q}(] a, b\right]\right)$ by equation (5).

REMARK 3.5. By changing the inequalities suitably, the proof also shows

$$
{ }^{\perp} \mathscr{Q}(>b) \cap \mathscr{Q}(<a)^{\perp}=\mathscr{Q}([a, b]) .
$$

The next lemma makes the formal connection to co-t-structures. It is analogous to the last part of $[\mathbf{4}$, Section 3].

LEMMA 3.6. If $\mathscr{Q}$ is a co-slicing of $\mathrm{T}$, then $(\mathscr{Q}(\leq 1), \mathscr{Q}(>1))$ is a bounded co-tstructure in $\mathrm{T}$ with co-heart $\mathscr{Q}(] 0,1])$.

Proof. The co-t-structure: We must check Definition 2.1. The subcategories $\mathscr{Q}(\leq$ 1) and $\mathscr{Q}(>1)$ are full and closed under direct sums and summands by definition. Definition 2.1(i) follows from equation (1). Definition 2.1(ii) follows from Definition 3.1(ii). And Definition 2.1(iii) follows from Lemma 3.2.

Boundedness: Clear by Definition 3.1(i) and (iii).

The co-heart: In a co-t-structure $(A, B)$, we have $A={ }^{\perp} B$ and $B=A^{\perp}$ whence $\Sigma^{-1} \mathrm{~B}=\left(\Sigma^{-1} \mathrm{~A}\right)^{\perp}$, so the co-heart is $\mathrm{C}=\mathrm{A} \cap \Sigma^{-1} \mathrm{~B}={ }^{\perp} \mathrm{B} \cap\left(\Sigma^{-1} \mathrm{~A}\right)^{\perp}$. Inserting the co-t-structure of this lemma gives $\mathrm{C}=\mathscr{Q}(] 0,1])$ by Lemma 3.4.

REMARK 3.7. Let $\mathscr{Q}$ be a co-slicing of T and let $a<b \leq a+1$ in $\mathbb{R}$. Then

$$
\mathscr{Q}(] a, b])=\operatorname{add}\left(\bigcup_{\varphi \in] a, b]} \mathscr{Q}(\varphi)\right) .
$$


The inclusion $\supseteq$ is clear, and $\subseteq$ holds because the right-hand side is closed under extensions. Indeed, any extension between two of its objects is trivial because of Definition 3.1(i) and (ii).

REMARK 3.8. Let $\mathscr{Q}$ be a co-slicing of T. Lemma 3.6 and Remark 3.7 imply that

$$
\mathrm{C}=\operatorname{add}\left(\bigcup_{\varphi \in] 0,1]} \mathscr{Q}(\varphi)\right)
$$

is the co-heart of the bounded co-t-structure $(\mathscr{Q}(\leq 1), \mathscr{Q}(>1))$ in T. The group $\mathrm{K}_{0}^{\text {split }}(\mathrm{C})$ is free on a basis consisting of the isomorphism classes of indecomposable objects in $\bigcup_{\varphi \in] 0,1]} \mathscr{Q}(\varphi)$. The group is isomorphic to $\mathrm{K}_{0}(\mathrm{~T})$ by Proposition 2.4 so is finitely generated by assumption.

It follows that $\mathscr{Q}(\varphi) \neq 0$ for only finitely many $\varphi \in] 0,1]$, and $\mathscr{Q}(\varphi)$ has only finitely many isomorphism classes of indecomposable objects for each $\varphi \in] 0,1]$.

Combining with Definition 3.1(i) shows that there exists $0<\varepsilon_{0}<\frac{1}{2}$ such that within each interval $\left[\varphi_{0}-\varepsilon_{0}, \varphi_{0}+\varepsilon_{0}\right]$, there is at most one $\varphi$ with $\mathscr{Q}(\varphi) \neq 0$. Similarly, it follows that $\mathscr{Q}(\varphi)$ has only finitely many isomorphism classes of indecomposable objects for each $\varphi \in \mathbb{R}$.

4. The metric space of co-slicings. In [4, Section 6], the set of slicings of a triangulated category was turned into a metric space, and we do the same for the set of co-slicings. The formula in the following definition is due to [4, Lemma 6.1].

Definition 4.1. If $\mathscr{Q}$ and $\mathscr{R}$ are co-slicings of T, then we set

$$
d(\mathscr{Q}, \mathscr{R})=\inf \{\varepsilon>0 \mid \mathscr{Q}(\varphi) \subseteq \mathscr{R}([\varphi-\varepsilon, \varphi+\varepsilon]) \text { for each } \varphi \in \mathbb{R}\} .
$$

REMARK 4.2. By Definition 3.1(i), we can replace $\mathbb{R}$ by $] 0,1]$ in the formula without changing the value of $d(\mathscr{Q}, \mathscr{R})$.

Proposition 4.3. The function d is a metric on the set of co-slicings of $\mathrm{T}$.

Proof. (i) $d(\mathscr{Q}, \mathscr{R})<\infty$ : By Remark 3.8, the subcategory $\mathscr{Q}(\varphi)$ is non-zero for only finitely many $\varphi \in] 0,1]$, and for each $\varphi$, it has only finitely many isomorphism classes of indecomposable objects. Using Definition 3.1(iii), this implies that there is an $\varepsilon>0$ such that $\mathscr{Q}(\varphi) \subseteq \mathscr{R}([\varphi-\varepsilon, \varphi+\varepsilon])$ for each $\varphi \in] 0,1]$. Hence, $d(\mathscr{Q}, \mathscr{R}) \leq \varepsilon$ by Remark 4.2.

(ii) $d(\mathscr{Q}, \mathscr{R})=d(\mathscr{R}, \mathscr{Q})$ : Given $\varepsilon>0$, by symmetry, it is enough to show that if $\mathscr{Q}(\varphi) \subseteq \mathscr{R}([\varphi-\varepsilon, \varphi+\varepsilon])$ for each $\varphi$, then $\mathscr{R}(\varphi) \subseteq \mathscr{Q}([\varphi-\varepsilon, \varphi+\varepsilon])$ for each $\varphi$. By Definition 3.1(ii), the condition $\mathscr{Q}(\varphi) \subseteq \mathscr{R}([\varphi-\varepsilon, \varphi+\varepsilon])$ for each $\varphi$ implies that $\mathrm{T}(\mathscr{R}(\varphi), \mathscr{Q}(>\varphi+\varepsilon))=0$ for each $\varphi$. That is,

$$
\mathscr{R}(\varphi) \subseteq{ }^{\perp} \mathscr{Q}(>\varphi+\varepsilon) \text { for each } \varphi
$$

Similarly, the condition implies

$$
\mathscr{R}(\varphi) \subseteq \mathscr{Q}(<\varphi-\varepsilon)^{\perp} \text { for each } \varphi .
$$

Together these inclusions imply $\mathscr{R}(\varphi) \subseteq \mathscr{Q}([\varphi-\varepsilon, \varphi+\varepsilon])$ for each $\varphi$ by Remark 3.5. 
(iii) $d(\mathscr{Q}, \mathscr{S}) \leq d(\mathscr{Q}, \mathscr{R})+d(\mathscr{R}, \mathscr{S})$ : If $d(\mathscr{Q}, \mathscr{R})=x$ and $d(\mathscr{R}, \mathscr{S})=y$, then there are inclusions $\mathscr{Q}(\varphi) \subseteq \mathscr{R}([\varphi-x-\delta, \varphi+x+\delta])$ and $\mathscr{R}(\varphi) \subseteq \mathscr{S}([\varphi-y-\delta, \varphi+y+$ $\delta])$ for each $\varphi \in \mathbb{R}$ and $\delta>0$. They clearly imply $\mathscr{Q}(\varphi) \subseteq \mathscr{S}([\varphi-(x+y)-2 \delta, \varphi+$ $(x+y)+2 \delta])$ whence $d(\mathscr{Q}, \mathscr{S}) \leq x+y$.

(iv) $d(\mathscr{Q}, \mathscr{R})=0 \Rightarrow \mathscr{Q}=\mathscr{R}$ : Let $q \in \mathscr{Q}(\varphi)$ be given. When $d(\mathscr{Q}, \mathscr{R})=0$, then $q \in$ $\mathscr{R}([\varphi-\varepsilon, \varphi+\varepsilon])$ for each $\varepsilon>0$. This implies $q \in \perp \mathscr{R}(>\varphi) \cap \mathscr{R}(<\varphi)^{\perp}$ by Definition 3.1 (ii) whence Remark 3.5 gives $q \in \mathscr{R}([\varphi, \varphi])=\mathscr{R}(\varphi)$. So, $\mathscr{Q}(\varphi) \subseteq \mathscr{R}(\varphi)$ and the opposite inclusion holds by symmetry.

5. Co-stability functions. This section introduces co-stability functions and the split Harder-Narasimhan property. They are analogues of the stability functions and the Harder-Narasimhan property of [4, Section 2], and will permit us to show that the co-stability manifold is divided into subsets corresponding to bounded co-t-structures; see Remark 8.5.

Definition 5.1. A co-stability function on an additive category $\mathrm{S}$ is a group homomorphism

$$
Z: \mathrm{K}_{0}^{\text {split }}(\mathrm{S}) \rightarrow \mathbb{C}
$$

such that $Z(s) \in H$ for each object $s \neq 0$, where

$$
H=\{r \exp (i \pi \varphi) \mid 0<r, 0<\varphi \leq 1\}
$$

is the strict upper half plane.

The phase $\varphi(s)$ of an object $s \supsetneqq 0$ is the unique element in $] 0,1]$ for which $Z(s)=$ $r \exp (i \pi \varphi(s))$.

We need a split version of Harder-Narasimhan theory so would like to define an object $s \neq 0$ to be $Z$-semistable if $s \cong s^{\prime} \oplus s^{\prime \prime}$ with $s^{\prime} \neq 0$ implies that $\varphi\left(s^{\prime}\right) \leq \varphi(s)$. However, this is equivalent to the following definition.

DEFINITION 5.2. Let $Z$ be a co-stability function on an additive category S. An object $s \in \mathrm{S}$ with $s \neq 0$ is called $Z$-semistable if $s \cong s^{\prime} \oplus s^{\prime \prime}$ with $s^{\prime} ¥ 0$ implies that $\varphi\left(s^{\prime}\right)=\varphi(s)$.

If $\mathrm{S}$ is Krull-Schmidt, then $s \in \mathrm{S}$ with $s \neq 0$ is $Z$-semistable if and only if its indecomposable direct summands have the same phase.

Definition 5.3. A co-stability function $Z$ on an additive category $S$ is said to have the split Harder-Narasimhan property if it satisfies the following:

(i) If $s_{1}, s_{2} \in \mathrm{S}$ with $s_{1}, s_{2} \not 0$ are $Z$-semistable with $\varphi\left(s_{1}\right)<\varphi\left(s_{2}\right)$, then we have $\mathrm{S}\left(s_{1}, s_{2}\right)=0$.

(ii) Each $s \in \mathrm{S}$ with $s \neq 0$ can be written $s \cong s_{1} \oplus \cdots \oplus s_{p}$ with the $s_{i}$ being $Z$ semistable and $\varphi\left(s_{1}\right)<\cdots<\varphi\left(s_{p}\right)$.

If $\mathrm{S}$ is Krull-Schmidt, then (ii) is vacuous but (i) is usually not.

6. Co-stability conditions. This section introduces co-stability conditions and proves a separation result in Proposition 6.2. We also show the precise relationship between co-stability conditions and co-t-structures in Proposition 6.3. These results are analogues of [4, Lemma 6.4] and [4, Proposition 5.3]. 
Definition 6.1. A co-stability condition on $\mathrm{T}$ is a pair $(Z, \mathscr{Q})$, where $Z: \mathrm{K}_{0}(\mathrm{~T}) \rightarrow$ $\mathbb{C}$ is a group homomorphism and $\mathscr{Q}$ a co-slicing of T such that

$$
q \in \mathscr{Q}(\varphi), q ¥ 0 \Rightarrow Z(q)=m(q) \exp (i \pi \varphi),
$$

with $m(q)>0$.

Proposition 6.2. If $(Z, \mathscr{Q})$ and $(Z, \mathscr{R})$ are co-stability conditions in $\mathrm{T}$ and $d(\mathscr{Q}, \mathscr{R})<\frac{1}{2}$, then $\mathscr{Q}=\mathscr{R}$.

Proof. When $d(\mathscr{Q}, \mathscr{R})<\frac{1}{2}$ holds, Definition 4.1 implies that there is $\varepsilon<\frac{1}{2}$ such that $\mathscr{Q}(\varphi) \subseteq \mathscr{R}(] \varphi-\varepsilon, \varphi+\varepsilon])$ for each $\varphi$. That is,

$$
\mathscr{Q}(\varphi) \subseteq \operatorname{add}\left(\bigcup_{\psi \in] \varphi-\varepsilon, \varphi+\varepsilon]} \mathscr{R}(\psi)\right)
$$

for each $\varphi$ by Remark 3.7. So, if $q \in \operatorname{ind} \mathscr{Q}(\varphi)$, then $q \in \operatorname{ind} \mathscr{R}(\psi)$ for a $\psi \in$ ]$\varphi-\varepsilon, \varphi+\varepsilon]$. Since $(Z, \mathscr{Q})$ and $(Z, \mathscr{R})$ are co-stability conditions, we get $Z(q)=$ $m(q) \exp (i \pi \varphi)$ and $Z(q)=m^{\prime}(q) \exp (i \pi \psi)$ with $m(q), m\left(q^{\prime}\right)>0$, and then $\psi=\varphi$ since $\varepsilon<\frac{1}{2}$. Hence, $q \in$ ind $\mathscr{R}(\varphi)$ and we learn $\mathscr{Q}(\varphi) \subseteq \mathscr{R}(\varphi)$. The opposite inclusion holds by symmetry.

Proposition 6.3. Giving a co-stability condition on $\mathrm{T}$ is equivalent to giving a bounded co-t-structure in $\mathrm{T}$ and a co-stability function on its co-heart which has the split Harder-Narasimhan property.

Proof. We describe how to map back and forth.

(i) Let $(Z, \mathscr{Q})$ be a co-stability condition on T. Then $(\mathscr{Q}(\leq 1), \mathscr{Q}(>1))$ is a bounded co-t-structure in $\mathrm{T}$ by Lemma 3.6. If $\mathrm{C}$ is the co-heart, then Proposition 2.4 gives an isomorphism $\mathrm{K}_{0}^{\text {split }}(\mathrm{C}) \rightarrow \mathrm{K}_{0}(\mathrm{~T})$ so $Z$ can be viewed as a group homomorphism $Z: \mathrm{K}_{0}^{\text {split }}(\mathrm{C}) \rightarrow \mathbb{C}$. This is a co-stability function on $\mathrm{C}$ which has the split HarderNarasimhan property.

(ii) Conversely, let $(A, B)$ be a bounded co-t-structure in $T$ with co-heart $C$, and let $Z$ be a co-stability function on $C$ which has the split Harder-Narasimhan property. Proposition 2.4 means that $Z$ can be viewed as a group homomorphism $Z: \mathrm{K}_{0}(\mathrm{~T}) \rightarrow \mathbb{C}$. For $0<\varphi \leq 1$, let $\mathscr{Q}(\varphi)$ be the full subcategory of $\mathrm{C}$ consisting of all objects which are isomorphic to 0 and all objects which are $Z$-semistable of phase $\varphi$; extend to other values of $\varphi$ by setting $\mathscr{Q}(\varphi+1)=\Sigma \mathscr{Q}(\varphi)$. Then $(Z, \mathscr{Q})$ is a co-stability condition.

7. Two triangle lemmas. The following two lemmas are easy consequences of the octahedral axiom and we omit the proofs:

LEMMA 7.1. Consider the following diagram in T consisting of two distinguished triangles.

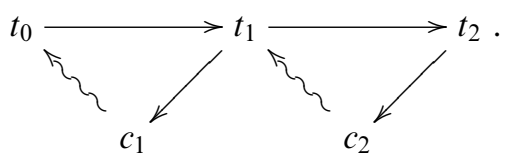


If $\mathrm{T}\left(c_{2}, \Sigma c_{1}\right)=0$, then there is a distinguished triangle

$$
t_{0} \longrightarrow t_{2} \longrightarrow c_{1} \oplus c_{2} \text {. }
$$

LEMMA 7.2. Consider the following distinguished triangle in $\mathrm{T}$.

$$
t_{0} \longrightarrow t_{2} \longrightarrow c_{1} \oplus c_{2} \text {. }
$$

Then there is the following diagram consisting of two distinguished triangles:

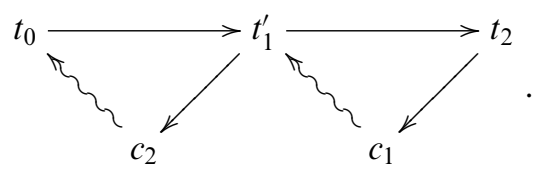

8. The co-stability manifold. This section proves a deformation result in Proposition 8.4; it is an analogue of [4, Theorem 7.1]. As in [4], by combining with a separation result, in our case Proposition 6.2, one obtains a manifold as a formal consequence. We formulate this as Theorem 8.3 which contains Theorem A.

An important ingredient is the following technical condition on separation which plays a role analogous to local finiteness in [4].

Definition 8.1. A co-slicing $\mathscr{Q}$ of $\mathrm{T}$ is said to satisfy condition (S) if

$$
q_{1}, q_{2} \in \operatorname{ind} \mathscr{Q}(\varphi), q_{1} ¥ q_{2} \Rightarrow \mathrm{T}\left(q_{1}, q_{2}\right)=0
$$

for each $\varphi$.

Let us write $\mathrm{K}_{0}(T)^{*}=\operatorname{Hom}_{\mathbb{Z}}\left(\mathrm{K}_{0}(\mathrm{~T}), \mathbb{C}\right)$. Since $\mathrm{K}_{0}(\mathrm{~T})$ is finitely generated, $\mathrm{K}_{0}(\mathrm{~T})^{*}$ is a finite-dimensional vector space over $\mathbb{C}$; it can be equipped with the usual topology. Let Coslice(T) denote the set of co-slicings of $\mathrm{T}$ satisfying condition (S); it is a metric space by Proposition 4.3 so in particular a topological space. Consider the product space $\mathrm{K}_{0}(\mathrm{~T})^{*} \times \operatorname{Coslice}(\mathrm{T})$.

DEFINITION 8.2. The co-stability manifold of $\mathrm{T}$ is the topological subspace

$$
\operatorname{Costab}(\mathrm{T}) \subseteq \mathrm{K}_{0}(\mathrm{~T})^{*} \times \operatorname{Coslice}(\mathrm{T}),
$$

consisting of co-stability conditions $(Z, \mathscr{Q})$.

The definition is motivated by the following theorem.

THEOREM 8.3. The topological space $\operatorname{Costab}(\mathrm{T})$ is a topological manifold which, if non-empty, has dimension $2 \cdot \operatorname{rankK}_{0}(\mathrm{~T})$.

As mentioned, this is a formal consequence of results on separation and deformation, which imply that the canonical map $\operatorname{Costab}(\mathrm{T}) \rightarrow \mathrm{K}_{0}(\mathrm{~T})^{*}$ given by $(Z, \mathscr{Q}) \mapsto Z$ is a local homeomorphism. In our case, separation is by Proposition 6.2 , while deformation takes the following form.

Proposition 8.4. Let $(Z, \mathscr{Q}) \in \operatorname{Costab}(\mathrm{T})$ be given and let $0<\varepsilon_{0}<\frac{1}{2}$ be as in Remark 3.8. 
Assume that $0<\varepsilon \leq \varepsilon_{0}$ and $W \in \mathrm{K}_{0}(\mathrm{~T})^{*}$ satisfies

$$
|W(q)-Z(q)|<\sin (\pi \varepsilon)|Z(q)|,
$$

for each $q \in \mathscr{Q}(\varphi)$ with $q ¥ 0$ and $\varphi \in \mathbb{R}$.

Then there is $(W, \mathscr{R}) \in \operatorname{Costab}(\mathrm{T})$ such that $d(\mathscr{Q}, \mathscr{R})<\varepsilon$.

Proof. For $\psi \in \mathbb{R}$, we define $\mathscr{R}(\psi)$ as the full subcategory of $\mathrm{T}$ which is closed under direct sums and summands and has the following indecomposable objects:

$$
\text { ind } \mathscr{R}(\psi)=\left\{\begin{array}{l|l}
q \in \operatorname{ind} \mathscr{Q}(\varphi) & \begin{array}{l}
\psi-\varepsilon<\varphi<\psi+\varepsilon \\
W(q)=m^{\prime}(q) \exp (i \pi \psi) \text { with } m^{\prime}(q)>0
\end{array}
\end{array}\right\}
$$

We will show that $\mathscr{R}$ is a co-slicing satisfying condition (S).

Definition 3.1(i) is clearly satisfied by $\mathscr{R}$.

Definition 3.1(ii) and condition (S): Let $r_{j} \in \operatorname{ind} \mathscr{R}\left(\psi_{j}\right)$ for $j=1,2$ and assume either $\psi_{1}<\psi_{2}$ (for Definition 3.1(ii)) or $\psi_{1}=\psi_{2}$ and $r_{1} \not \approx r_{2}$ (for condition (S)). By definition, we have $r_{j} \in \operatorname{ind} \mathscr{Q}\left(\varphi_{j}\right)$ with

$$
\psi_{j}-\varepsilon<\varphi_{j}<\psi_{j}+\varepsilon \text { for } j=1,2
$$

We split into three cases.

$\varphi_{1}<\varphi_{2}$ : Then $\mathrm{T}\left(r_{1}, r_{2}\right)=0$ by Definition 3.1(ii) for $\mathscr{Q}$.

$\varphi_{1}=\varphi_{2}$ : There are two possibilities. First, we may have $\psi_{1}=\psi_{2}$. Then $r_{1} \neq r_{2}$ by assumption whence $\mathrm{T}\left(r_{1}, r_{2}\right)=0$ by condition (S) for $\mathscr{Q}$. Secondly, we may have $\psi_{1}<$ $\psi_{2}$. We also have $\psi_{2}<\psi_{1}+2 \varepsilon$ by the inequalities (6), and $2 \varepsilon<2 \varepsilon_{0}<1$, so $W\left(r_{j}\right)=$ $m^{\prime}\left(r_{j}\right) \exp \left(i \pi \psi_{j}\right)$ implies that $W\left(r_{1}\right) \neq W\left(r_{2}\right)$. But then $r_{1} \not r_{2}$ whence $\mathrm{T}\left(r_{1}, r_{2}\right)=0$ by condition (S) for $\mathscr{Q}$.

$\varphi_{1}>\varphi_{2}$ : The inequality (6) also gives $\varphi_{1}<\varphi_{2}+2 \varepsilon$, so $\varphi_{1}$ is certainly in the interval $\left[\varphi_{2}, \varphi_{2}+2 \varepsilon_{0}\right]$ and so is $\varphi_{2}$. But by Remark 3.8, each closed interval of length $2 \varepsilon_{0}$ contains at most one $\varphi$ with $\mathscr{Q}(\varphi) \neq 0$. This gives a contradiction with $\varphi_{1} \neq \varphi_{2}$ and $r_{j} \in \operatorname{ind} \mathscr{Q}\left(\varphi_{j}\right)$.

Definition 3.1(iii): We start with an observation. If $q \in$ ind $\mathscr{Q}(\varphi)$ with $q ¥ 0$, then the inequality in the proposition implies $W(q)=m^{\prime}(q) \exp (i \pi \psi)$ with $m^{\prime}(q)>0$ and $\psi$ satisfying $\psi-\varepsilon<\varphi<\psi+\varepsilon$, whence $q \in \mathscr{R}(\psi)$.

Given $t \in \mathrm{T}$, we must establish a diagram as in Definition 3.1(iii) for $\mathscr{R}$. This is trivial for $t \cong 0$ so suppose $t \not 0$. Using that $\mathscr{Q}$ is a co-slicing, pick a diagram as in Definition 3.1(iii). We can assume $q_{j} \not 0$ for each $j$. Using Lemma 7.2, each distinguished triangle in the diagram can be refined to a sequence of distinguished triangles with indecomposable third term. This gives a diagram

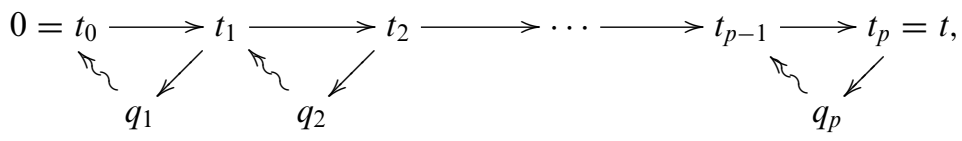

consisting of distinguished triangles where $q_{j} \in \operatorname{ind} \mathscr{Q}\left(\varphi_{j}\right)$.

By the above observation, we have $q_{j} \in \mathscr{R}\left(\psi_{j}\right)$ for each $j$ for certain $\psi_{j} \in \mathbb{R}$. Suppose that $\psi_{j}>\psi_{j+1}$ for some $j$. Then $\mathrm{T}\left(q_{j+1}, \Sigma q_{j}\right)=0$ by Definition 3.1(i) and (ii), which we 
have already shown for $\mathscr{R}$. So Lemmas 7.1 and 7.2 imply that in diagram (7), the part

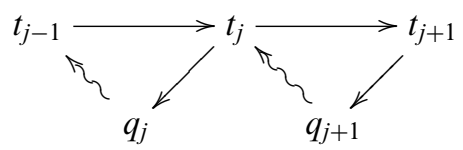

can be replaced with

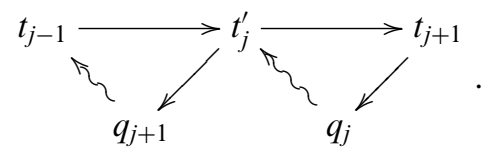

Repeating this procedure reorders the $q_{j}$ according to non-decreasing values of $\psi_{j}$. That is, it turns diagram (7) into a diagram

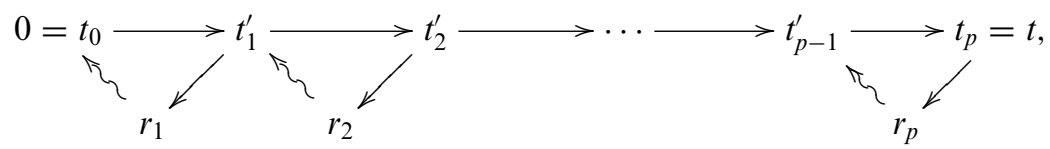

consisting of distinguished triangles, where $r_{j} \in \operatorname{ind} \mathscr{R}\left(\psi_{j}\right)$ and $\psi_{1} \leq \cdots \leq \psi_{p}$. Neighbouring objects $r_{j}$ and $r_{j+1}$ with $\psi_{j}=\psi_{j+1}$ have $\mathrm{T}\left(r_{j+1}, \Sigma r_{j}\right)=0$, again by Definition 3.1(i) and (ii), so $r_{j}$ and $r_{j+1}$ can be collected using Lemma 7.1. This finally gives a diagram as in Definition 3.1(iii) for $\mathscr{R}$.

To complete the proof, we must show that $(W, \mathscr{R})$ is a co-stability condition and that $d(\mathscr{Q}, \mathscr{R})<\varepsilon$. The former is clear by the definition of $\mathscr{R}$. For the latter, note that by Remark 3.8, if $\psi$ is given, then there are only finitely many $\varphi$ with $\psi-\varepsilon<\varphi<\psi+\varepsilon$ and $\mathscr{Q}(\varphi) \neq 0$. Hence, there is an $\varepsilon^{\prime}<\varepsilon$ such that it makes no difference to replace $\varepsilon$ by $\varepsilon^{\prime}$ in the definition of ind $\mathscr{R}(\psi)$, and so $\mathscr{R}(\psi) \subseteq \mathscr{Q}\left(\left[\psi-\varepsilon^{\prime}, \psi+\varepsilon^{\prime}\right]\right)$. This applies to each of the finitely many $\psi \in] 0,1]$ for which $\mathscr{R}(\psi) \neq 0$; see Remark 3.8 again. But then $d(\mathscr{Q}, \mathscr{R})<\varepsilon$ by Remark 4.2.

Remark 8.5. By Proposition 6.3, each point $(Z, \mathscr{Q}) \in \operatorname{Costab}(T)$ corresponds to a pair consisting of a bounded co-t-structure in $\mathrm{T}$ and a co-stability function on its co-heart, which has the split Harder-Narasimhan property. In particular, Costab(T) is divided into subsets corresponding to different co-t-structures in $\mathrm{T}$.

9. Two group actions on the co-stability manifold. Like the stability manifold, the co-stability manifold admits commuting continuous left and right actions of the groups $\operatorname{Aut}(T)$ and $\widetilde{G L}^{+}(2, \mathbb{R})$, where $\operatorname{Aut}(T)$ is the group of equivalence classes of triangulated autoequivalences of $\mathrm{T}$ and $\widetilde{\mathrm{GL}}^{+}(2, \mathbb{R})$ is the universal cover of $\mathrm{GL}^{+}(2, \mathbb{R})$, the group of $2 \times 2$ real matrices with positive determinant. Indeed, we can just copy the formulae from [4, Lemma 8.2] as follows.

For $F \in \operatorname{Aut}(\mathrm{T})$ and $(Z, \mathscr{Q}) \in \operatorname{Costab}(\mathrm{T})$, set

$$
F \cdot(Z, \mathscr{Q})=\left(Z \circ[F]^{-1}, \mathscr{Q}^{\prime}\right),
$$

where $[F] \in \operatorname{AutK}_{0}(\mathrm{~T})$ is induced by $F$ and $\mathscr{Q}^{\prime}(\varphi)$ is the closure under isomorphisms of $F(\mathscr{Q}(\varphi))$. 
For $\widetilde{G L}^{+}(2, \mathbb{R})$, we use the same description as in [4, Section 8], so an element is a pair $(T, f)$ where $T: \mathbb{R}^{2} \rightarrow \mathbb{R}^{2}$ is an orientation-preserving linear map and $f: \mathbb{R} \rightarrow \mathbb{R}$ is an increasing map satisfying $f(x+1)=f(x)+1$, such that the induced maps on $\left(\mathbb{R}^{2} \backslash 0\right) / \mathbb{R}_{>0}$ and $\mathbb{R} / 2 \mathbb{Z}$ are the same when these spaces are identified with $S^{1}$. For $(T, f) \in \widetilde{\mathrm{GL}}^{+}(2, \mathbb{R})$ and $(Z, \mathscr{Q}) \in \operatorname{Costab}(\mathrm{T})$, set

$$
(Z, \mathscr{Q}) \cdot(T, f)=\left(T^{-1} \circ Z, \mathscr{Q}^{\prime \prime}\right),
$$

where $\mathscr{Q}^{\prime \prime}(\varphi)=\mathscr{Q}(f(\varphi))$.

10. Example: The compact derived category of $k[X] /\left(X^{2}\right)$. Let $k$ be an algebraically closed field. The compact derived category $\mathrm{D}^{\mathrm{c}}\left(k[X] /\left(X^{2}\right)\right)$ of the dual numbers over $k$ is the special case $w=0$ of $\mathrm{U}$ in the next theorem, so Theorem $\mathrm{B}$ in Section 1 follows.

THEOREM 10.1. Let $w \leq 0$ be an integer and let $\mathrm{U}$ be a $k$-linear algebraic triangulated category with split idempotents which is classically generated by a w-spherical object; see [5].

The stability manifold of $\bigcup$ is the empty set. The co-stability manifold of $\bigcup$ is $\mathbb{C}$.

Proof. By [5, Theorem A], the category $U$ has no non-trivial t-structures, hence no bounded t-structures. It follows by [4, Proposition 5.3] that it has no stability conditions, so the stability manifold is the empty set.

By $[5$, Theorem $A]$ again, the category $U$ has a canonical co-t-structure $(A, B)$, and the non-trivial co-t-structures in $U$ are precisely the (de)suspensions $\left(\Sigma^{j} A, \Sigma^{j} B\right)$ for $j \in \mathbb{Z}$. The explicit description of the canonical co-t-structure in [5, Section 4.e] shows that each of the (de)suspensions is bounded. It also shows that the co-heart $\mathrm{C}$ is equal to $\operatorname{add}(c)$ for a certain indecomposable object $c$. Hence, the co-heart of $\left(\Sigma^{j} \mathrm{~A}, \Sigma^{j} \mathrm{~B}\right)$ is $\Sigma^{j} \mathrm{C}=\operatorname{add}\left(\Sigma^{j} c\right)$

Combining this with Proposition 6.3 shows that giving a co-stability condition on $\mathrm{U}$ is equivalent to giving two pieces of data: (i) an integer $j$ specifying a bounded co-tstructure ( $\left.\Sigma^{j} \mathrm{~A}, \Sigma^{j} \mathrm{~B}\right)$ and (ii) an element $z$ of the strict upper half plane $H$ specifying a co-stability function on the co-heart as follows:

$$
Z: \mathrm{K}_{0}^{\text {split }}\left(\Sigma^{j} \mathrm{C}\right) \rightarrow \mathbb{C}, \quad Z\left(\Sigma^{j} c\right)=z
$$

The split Harder-Narasimhan property holds for $Z$ because $\Sigma^{j} \mathrm{C}$ has only one isomorphism class of indecomposable objects.

By the proof of Proposition 6.3, these data correspond to the following costability condition $(Z, \mathscr{Q})$ : By means of Proposition 2.4 , the above $Z$ is viewed as a group homomorphism $Z: \mathrm{K}_{0}(\mathrm{U}) \rightarrow \mathbb{C}$; it still satisfies $Z\left(\Sigma^{j} c\right)=z$. And writing $z=r \exp (i \pi \varphi)$ with $r>0, \varphi \in] 0,1]$, the co-slicing $\mathscr{Q}$ is given by $\mathscr{Q}(\varphi)=\operatorname{add}\left(\Sigma^{j} c\right)$ and $\mathscr{Q}$ equal to zero on the rest of the interval $] 0,1]$.

This co-stability condition can also be described by giving the unique $\varphi_{0} \in \mathbb{R}$ for which $\mathscr{Q}\left(\varphi_{0}\right)=\operatorname{add}(c)$, along with the complex number $Z(c)=z_{0}$ which has the form $z_{0}=s \exp \left(i \pi \varphi_{0}\right)$ for some $s>0$. Abusing notation, we write $(Z, \mathscr{Q})=\left(z_{0}, \varphi_{0}\right)$.

Each co-stability condition clearly satisfies condition (S).

Let $G$ be the closed subgroup of $\widetilde{\mathrm{GL}}^{+}(2, \mathbb{R})$ consisting of elements $(T, f)$, where $T$ is the composition of a rotation and a scaling by a positive real number. Note that $f(x)=x+a$, where $a$ is a real number determined modulo $2 \mathbb{Z}$ by $T$. Since $G$ is a 
subgroup of $\widetilde{G L}^{+}(2, \mathbb{R})$, it acts continuously on $\operatorname{Costab}(U)$ by Section 9 . The action is given by

$$
\left(z_{0}, \varphi_{0}\right) \cdot(T, f)=\left(T^{-1} z_{0}, \varphi_{0}-a\right)
$$

It is easy to see that the action is free and transitive, so Costab $(U)$ is homeomorphic to $G$.

However, $G$ is simply connected and $2 \mathbb{Z}$ can be viewed as the discrete subgroup consisting of the elements (id, $x \mapsto x+y$ ) for $y \in 2 \mathbb{Z}$. Hence, $G$ is the universal covering group of $G / 2 \mathbb{Z}$, but $G / 2 \mathbb{Z}$ can be identified with the subgroup of $\mathrm{GL}^{+}(2, \mathbb{R})$ consisting of transformations $T$ which are the composition of a rotation and a scaling by a positive real number. Hence, $G / 2 \mathbb{Z}$ is homeomorphic to $\mathbb{C} \backslash 0$, so $G$ is homeomorphic to the universal cover which is $\mathbb{C}$.

\section{Example: The compact derived category of $k A_{2}$. Why Condition (S) is necessary.} This section shows that without condition (S) of Definition 8.1, the conclusion of our deformation result Proposition 8.4 fails.

Let $k$ be an algebraically closed field. The Auslander-Reiten quiver of the compact derived category $\mathrm{V}=\mathrm{D}^{\mathrm{c}}\left(k A_{2}\right)$ is $\mathbb{Z} A_{2}$. Let $x$ and $y$ be consecutive indecomposable objects on the quiver, then $\mathrm{K}_{0}(\mathrm{~V})$ is free on the generators $[x]$ and $[y]$.

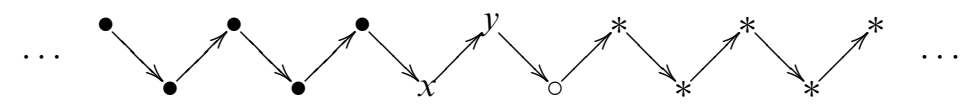

Let $A$ denote add of the indecomposable objects forming the left-hand part of the quiver ending at $y$; some of them are marked with bullets in the sketch. Let B denote add of the indecomposable objects forming the right-hand part of the quiver, starting with the asterisks in the sketch. It is not hard to check that $(A, B)$ is a bounded co-t-structure in $\mathrm{V}$. The co-heart is $\mathrm{C}=\mathrm{A} \cap \Sigma^{-1} \mathrm{~B}=\operatorname{add}(x, y)$.

Define a co-stability function $Z: \mathrm{K}_{0}^{\text {split }}(\mathrm{C}) \rightarrow \mathbb{C}$ by $Z(x)=Z(y)=\exp \left(i \pi \frac{1}{2}\right)$; it clearly has the split Harder-Narasimhan property.

By the proof of Proposition 6.3, the data (A, B) and $Z$ correspond to the following co-stability condition $(Z, \mathscr{Q})$ : By means of Proposition 2.4, the above $Z$ is viewed as a group homomorphism $Z: \mathrm{K}_{0}(\mathrm{~V}) \rightarrow \mathbb{C}$; it still satisfies $Z(x)=Z(y)=\exp \left(i \pi \frac{1}{2}\right)$. The co-slicing $\mathscr{Q}$ is given by $\mathscr{Q}\left(\frac{1}{2}\right)=\operatorname{add}(x, y)$ and $\mathscr{Q}(\varphi)=0$ for $\left.\left.\varphi \in\right] 0,1\right] \backslash \frac{1}{2}$.

Let $\varepsilon<\frac{1}{2}$ be given and let $W \in \mathrm{K}_{0}(\mathrm{~V})^{*}$ be the deformation of $Z$ defined by $W(x)=$ $\exp \left(i \pi \frac{1}{2}\right)$ and $W(y)=\cos (\pi \varepsilon) \exp \left(i \pi\left(\frac{1}{2}+\varepsilon\right)\right)$. This $W$ is chosen to satisfy two criteria: (i) compared to $Z$, it fixes $x$ but moves $y$ from phase $\frac{1}{2}$ to phase $\frac{1}{2}+\varepsilon$; (ii) it satisfies the inequality in Proposition 8.4 because of the factor $\cos (\pi \varepsilon)$.

PROPOSITION 11.1.

(i) Condition $(S)$ fails for $(Z, \mathscr{Q})$.

(ii) The conclusion of Proposition 8.4 fails for the deformation $W$. That is, there is no $(W, \mathscr{R}) \in \operatorname{Costab}(\mathrm{V})$ such that $d(\mathscr{Q}, \mathscr{R})<\varepsilon$.

Proof. (i) This is clear because $x, y \in \mathscr{Q}\left(\frac{1}{2}\right)$, while $\mathrm{V}(x, y) \neq 0$.

(ii) We show more than formulated, namely, there is no $(W, \mathscr{R}) \in \operatorname{Costab}(\mathrm{V})$ such that $d(\mathscr{Q}, \mathscr{R})<\frac{1}{2}$. For suppose that there is. Then we have $d(\mathscr{Q}, \mathscr{R})<\delta<\frac{1}{2}$ for some 
$\delta$ and this gives the first of the inclusions in the following formula:

$$
\left.\left.\operatorname{add}(x, y)=\mathscr{Q}\left(\frac{1}{2}\right) \subseteq \mathscr{R}\left(\left[\frac{1}{2}-\delta, \frac{1}{2}+\delta\right]\right) \subseteq \mathscr{R}(] 0,1\right]\right)=\operatorname{add}\left(\bigcup_{\psi \in] 0,1]} \mathscr{R}(\psi)\right)
$$

The last equality is by Remark 3.7 .

By Remark 3.8, the right-hand side of this formula is the co-heart of a bounded co-t-structure in V, so it follows from Proposition 2.4 that the right-hand side has precisely two isomorphism classes of indecomposable objects that must necessarily be the isomorphism classes of $x$ and $y$.

However, since $(W, \mathscr{R})$ is a co-stability condition, we have $W(r)=m^{\prime}(r) \exp (i \pi \psi)$ for $r \in \mathscr{R}(\psi)$ with $r \not 0$. The values $W(x)$ and $W(y)$ hence force $x \in \mathscr{R}\left(\frac{1}{2}\right)$ and $y \in$ $\mathscr{R}\left(\frac{1}{2}+\varepsilon\right)$. But this contradicts $\mathrm{V}(x, y) \neq 0$ by Definition 3.1(ii).

ACKnOwledgement. Part of this work was carried out while Jørgensen was visiting Hannover supported by the research priority programme SPP 1388 Darstellungstheorie of the Deutsche Forschungsgemeinschaft (DFG). He gratefully acknowledges the financial support through the grant HO 1880/4-1 held by Thorsten Holm.

\section{REFERENCES}

1. T. Aihara and O. Iyama, Silting mutation in triangulated categories, J. London Math. Soc. to appear, math.RT/1009.3370v2. doi: $10.1112 / \mathrm{jlms} / \mathrm{jdr} 055$

2. L. Avramov and S. Halperin, Through the looking glass: A dictionary between rational homotopy theory and local algebra, in Algebra, algebraic topology and their interactions (Roos J.-E., Editor), Lecture Notes in Mathematics, vol. 1183 (Springer, Berlin, 1986), 1-27.

3. M. V. Bondarko, Weight structures vs. t-structures; weight filtrations, spectral sequences, and complexes (for motives and in general), J. K-Theory 6 (2010), 387-504. $317-345$.

4. T. Bridgeland, Stability conditions on triangulated categories, Ann. Math. 166(2) (2007),

5. T. Holm, P. Jørgensen and D. Yang, Sparseness of t-structures and negative Calabi-Yau dimension in triangulated categories generated by a spherical object, Bull. Lond. Math. Soc., Preprint (2011). math.RT/1108.2195v1.

6. O. Mendoza, E. C. Saenz, V. Santiago and M. J. S. Salorio, Auslander-Buchweitz context and co-t-structures, Appl. Categorical Struct. Preprint (2010). math.CT/1002.4604v2. doi: 10.1007/s10485-011-9271-2

7. D. Pauksztello, Compact corigid objects in triangulated categories and co- $t$-structures, Cent. Eur. J. Math. 6 (2008), 25-42. 